\title{
Research on Integrity management system based on corrosion risk online monitoring (CROM)
}

\author{
HaiTao Lai ${ }^{1, a^{*}}$, FaGuo Tian $^{1}$, ZaiShun $\mathrm{Li}^{1}$, Shuzhen $\mathrm{Yu}^{1}$ and Bin Yang ${ }^{2}$ \\ ${ }^{1}$ Sulige Gas Field Research Center, PetroChina Changqing Oilfield Company \\ ${ }^{2}$ National Center for materials service safety, University of Science \& Technology Beijing \\ alht_cq@petrochina.com.cn
}

\begin{abstract}
Keywords: Integrity management system; corrosion risk; online monitoring
\end{abstract}
\begin{abstract}
This paper is proposed based on the risk of corrosion online monitoring (CROM) of oil and gas field station key equipment integrity management system, the key for the key equipment of oil and gas field station (industrial pipeline and equipment), through on-line monitoring technology on corrosion and continuous monitoring of key equipment service condition, obtain the evolution process of key equipment performance, evaluation the corrosion risk level of key equipment, prediction of critical equipment service life.
\end{abstract}

\section{Introduction}

In the field of petroleum and petrochemical industry, the concept of integrity management is used in long distance oil pipeline, and comes from the risk management of long distance pipeline of Europe and America in 1970s. However, with the rapid development of industry, an amalgamation of industry, integrity management concept is not limited in long-distance oil and gas pipeline. In the process equipment, even power machinery and equipment have carried out a lot of research and practical work. Therefore, the definition of oil and gas field station key equipment integrity management is mainly for industrial pipes outside the station, process and equipment to on-line monitoring of the main, accumulation of the key equipment of the service data and process data, risk identification and classification, risk model to build oil and gas field station key equipment, and can carry out the real-time and on-line updating model, guide the oil and gas station operation and maintenance. [1-2].

In the field of petroleum and petrochemical industry, the concept of integrity management is used in long distance oil pipeline, and the risk management of long distance pipeline from Europe and America in 1970s. However, with the rapid development of industry, an amalgamation of industry, integrity management concept is not limited in long-distance oil and gas pipeline, in the process equipment, even power machinery and equipment have carried out a lot of research and practical work. Therefore, the definition of oil and gas field station key equipment integrity management is mainly for industrial pipes outside the station, process and equipment to on-line monitoring of the main, accumulation of the key equipment of the service data and process data, risk identification and classification, risk model to build oil and gas field station key equipment, and can carry out the real-time and on-line updating model, guide the oil and gas station operation and maintenance. [3-4].

Therefore, this paper is proposed based on the risk of corrosion online monitoring (CROM) of oil and gas field station key equipment integrity management system, the key for the key equipment of oil and gas field station (industrial pipeline and equipment), through on-line monitoring technology on corrosion and continuous monitoring of key equipment service condition, obtain the evolution process of key equipment performance, evaluation the corrosion risk level of key equipment, prediction of critical equipment service life. 


\section{System Architecture and Online monitoring scheme}

The integrity management system based on corrosion risk on-line monitoring (CROM) mainly adopts five layers of distributed network architecture, that is, presentation layer, data persistence layer, service control layer, service layer and data source layer.

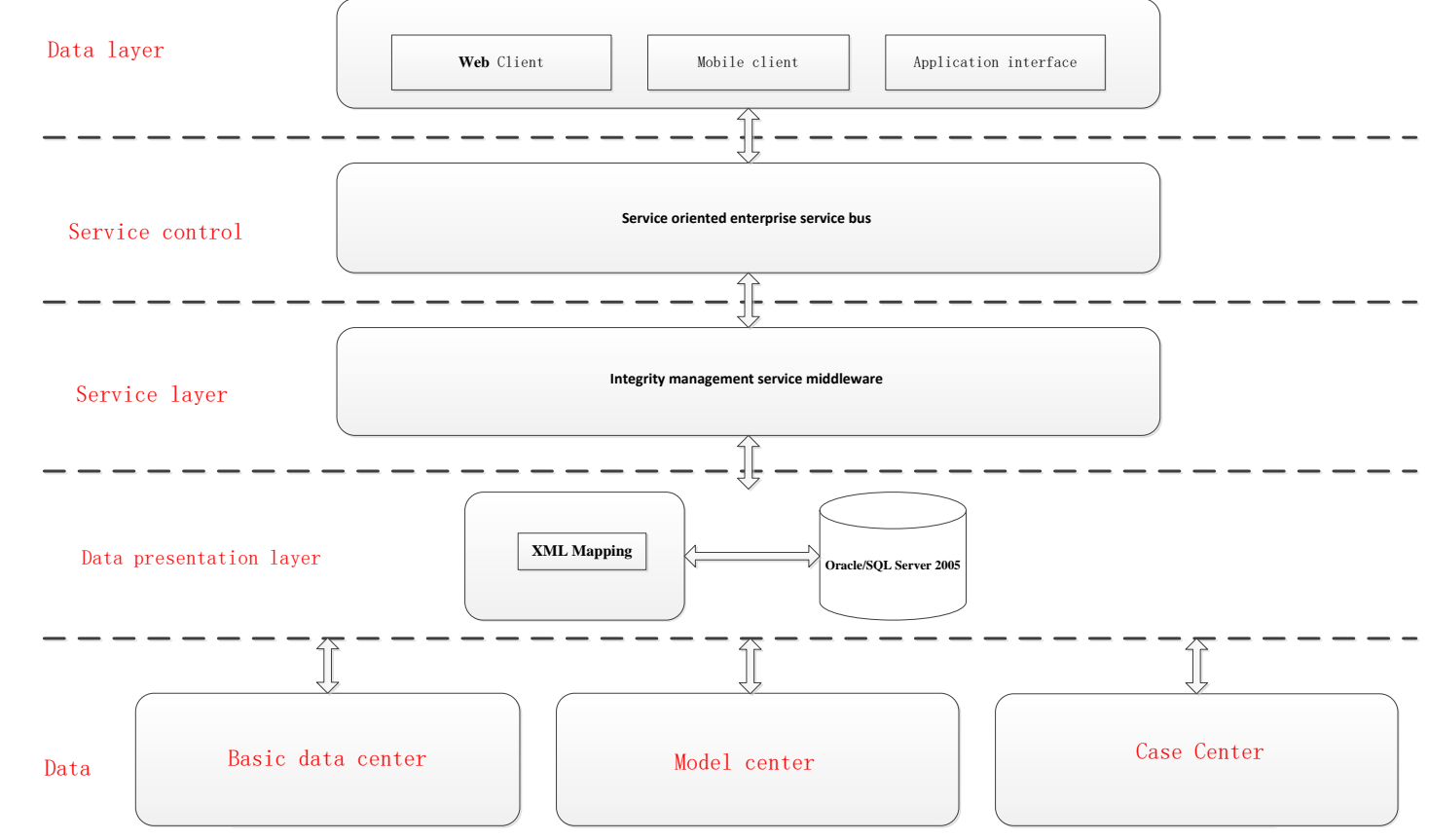

Fig. 1 Integrity management system architecture

1) Data layer. The system data provider in the integrity management system should include basic data, model and the case of three types of data, including basic data is accumulated through offline and online mode; model center includes risk model existing and accumulation model, has the characteristics of real-time on line update, some models can not only through the existing real-time correction the basic data accumulated, but also through data fitting based on some new models; case center is mainly through the accumulation of daily production in different production conditions and operation parameters under the formation of corrosion and repair risk of this case, as the basis of judgment provide evaluation basis for corrosion risk assessment model and evaluation.

2) Data persistence layer. Mainly responsible for the realization of the data layer is data persistence operation. Refers to the domain object permanently saved to the database; generalized understanding, "persistence" includes the various operations related to the database. Data Persistence technology is used to encapsulate the details of data access, for the majority of business logic to provide object-oriented API. Through Persistence technology can reduce the number of database data access to and increase the application program execution speed; high reusability of code can be completed most of the database operation; loose coupling, the persistence does not depend on the underlying database and the upper business logic implementation, when replacing the database just modify the configuration file without modifying the code. Its main operations include: preservation: the domain object permanently saved to the database; II updates: updates the state of the database domain object; delete (3): to delete a domain object from the database; 4 loading: according to the specific oid, the a domain object is loaded from the database into memory, query: according to the specific query conditions, to meet the conditions to the query one or a plurality of domain objects from the database loading internal storage.

3) Service layer is equivalent to the role of middleware, provides a universal interface cohesion said layer and data layer, hiding the details of the business logic layer, the internal need to organize business micro service and provide a more macro and for the presentation layer service logic and using contract interfaces exposed, package. Establishing a service layer in the development can be no need to pay attention to how the underlying architecture or the underlying occurrence of changes. 
4) Service control layer is a unified access system for all kinds of devices, terminals, third party system interface or standard interface. The service control layer provides a personalized interface or interface according to the terminal and the access authority to access the user.

5) Application layer to implement user login interface, including web client and mobile client and the third party application interface, and control the user application environment of different levels and different business areas, the sharing of information and resources to achieve upload and download.

The project as shown in Figure 2-3 shows the system architecture diagram, using a simplified three layered network architecture, the first layer is composed of wireless multi-channel ultrasonic sensor network wall thickness data acquisition layer; the second layer is formed by a relay node and sink node data transmission network, according to the different can be combined relay node and sink node, to form a more flattened data transmission network; the third layer is the remote online monitoring system, for users provide distributed access service, within the network to realize data transmission conditions of remote access.

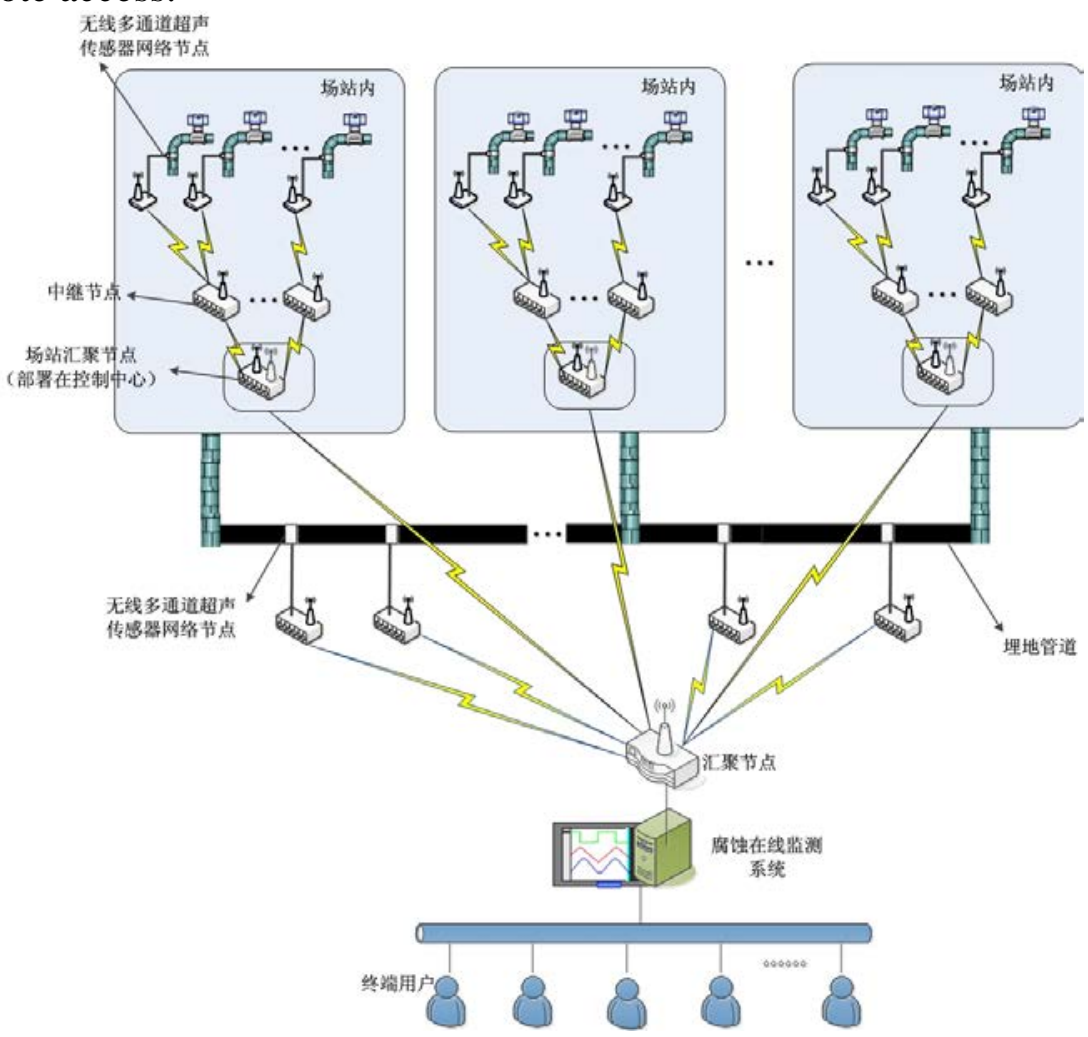

Fig. 2 Schematic diagram of system architecture

The main components of the system are as follows:

(1) Ultrasonic sensor: responsible for measuring the wall thickness of the pipe.

(2) wireless multi channels ultrasonic monitoring sensor network nodes: periodic collection of wall thickness data, according to the needs of users set the sampling cycle (the system to provide the sampling cycle include: once a day, three days a time, a week, a month at a time). At the same time, the wireless module sends the wall thickness data to the relay node or sink node. Using the battery power supply, regular Automatic wake-up work, complete the wall thickness data collection, after sending, automatically enter the sleep state;

(3) relay nodes: receiving from wireless multichannel ultrasonic sensor monitoring network node sends data of wall thickness, and is transmitted to the sink node, both can be designed as a battery powered, can also according to the site by power supply;

(4) sink node: receive the wall thickness data from the relay node, and send to the corrosion online monitoring system through the serial port, which is stored in the database. The sink node is connected to the server through the serial port, and the power supply is adopted; 
(5) of on-line corrosion monitoring system: provide wall thickness data browse, query and statistical analysis functions, also has an event alarm function, the wall thickness threshold set by the user, wireless multichannel ultrasonic monitoring sensor network node energy threshold alarm.

Each wireless multichannel ultrasonic monitoring sensor network nodes into eight channels or 4 channel according to the site design requirements. In this project by four channel design patterns, namely each wireless multichannel ultrasonic monitoring sensor network nodes can connect four ultrasonic sensors. The wall thickness data were collected by polling, and the wall thickness was measured in 4 measurement points, and the time was 30 seconds.

\section{Summary}

This paper combined with multi channels ultrasonic research and development of wireless multichannel ultrasonic sensor network wall thickness monitoring node thickness technology, wireless sensor network technology to design monitor, and practical application in oil and gas field station process pipe wall thickness on-line monitoring, running through the field to verify the accuracy and stability of the system, to fill the domestic blank. At the same time, the design of the five layer distributed based on the risk of corrosion online monitoring (CROM) of oil and gas field station key equipment integrity management system, the data acquisition is embodied in the concept of basic data center, the center of the model and case center.

\section{References}

[1] Mingxing Bai, Zhichao Zhang, Xiaofei Fu. A review on well integrity issues for CO2 geological storage and enhanced gas recovery [J], Renewable and Sustainable Energy Reviews, 2016, 59, 920-926

[2] R. Venkatesan, G. Vengatesan, N. Vedachalam, M. Arul Muthiah, R. Lavanya, M.A. Atmanand. Reliability assessment and integrity management of data buoy instruments used for monitoring the Indian Seas[J]. Applied Ocean Research, 2016, 54, 1-11.

[3] Hossam A. Kishawy, Hossam A. Gabbar. Review of pipeline integrity management practices [J]. International Journal of Pressure Vessels and Piping, 2010, 87(7):373-380

[4] Yousif Rahim, Ingbjørn Refsdal, Ron S. Kenett. The 5C model: A new approach to asset integrity management [J]. International Journal of Pressure Vessels and Piping, 2010, 87(2-3):88-93 